\title{
Continuous Media Streams Service Based on the Adaptive Buffer Sharing Policy
}

\author{
Yong Woon Park ${ }^{1}$ and Si Woong Jang ${ }^{2}$ \\ ${ }^{1}$ Department of Computer Science, Dong-eui Institute of Technology, san72, Yangjung Dong, \\ Pusanjin Ku, Pusan, Rep. of Korea \\ ywpark@dit.ac.kr \\ ${ }^{2}$ Department of Computer Science, Dong-eui University, san24, Kaya Dong, Pusanjin Ku, \\ Pusan, Rep. of Korea \\ swjang@hyomin.dongeui.ac.kr
}

\begin{abstract}
In this paper, a stream service strategy based on the adaptive buffer sharing and prefetching policy for the continuous media stream service is proposed. In the proposed caching policy, a stream is given one of the three service modes- direct, shared or object- with its requested object's access patterns and frequency. With direct mode, a stream is serviced directly from disk using its own read-ahead buffer, which is necessary to buffer the speed discrepancy between disk's transfer rate and user's playback rate for the requested stream. In shared mode, the interval between two consecutive streams accessing the same object is cached whereas in object mode, which could be set when the access frequency of the requested object exceeds a given threshold; an object is cached in its entirety. The service mode of each object could be changed dynamically with its access frequency.
\end{abstract}

\section{Introduction}

Traditionally, caching has been studied in the literature to reduce the cost of the internal device I/O by caching the frequently reused objects in the buffer memory. However, traditional caching policies employed for non-multimedia applications are not appropriate for multimedia applications[3]. So, in this paper, we propose a stream service strategy based on the adaptive caching policy where each stream is given one of the following three service modes - direct, shared or object - based on the access patterns and frequency of its requested object. In shared mode, the interval formed with the preceding stream accessing the same object is cached while in object mode, which could be set when the access frequency of the requested object exceeds a given threshold; an object is cached in its entirety. The processing mode of the object could be changed dynamically with its access frequency. Other streams that are not permitted to share buffer cache are run in direct mode; a stream is serviced directly from disk using its own read-ahead buffer, which is necessary to buffer the speed discrepancy between disk's transfer rate and user's playback rate for the requested stream. 


\section{Related Research}

Earlier works in multimedia buffer management have studied the buffer requirement for various disk-scheduling algorithms[1, 4, 6, 7]. In these works, the video data block is discarded once it has been transmitted to the clients and is not retained for subsequent reuse by other clients. In continuous media data caching policies in the literatures $[8,9]$, the buffer space is allocated to the interval between two consecutive streams of the same object. In this policy, a stream is cached as a function of the interval length and the size of the buffer cache. In this approach, the buffer blocks held by existing intervals are passed to the new interval formed by the new request if there is not so much free memory as the length of the newly formed interval. However, in this policy, in most cases, the succeeding streams must access the initial portion of the requested object from disks since those blocks are not guaranteed to be found in the buffer cache even if all subsequent accesses are serviced from the cache.

\section{Stream Service Strategy and Caching Policy}

\subsection{Data Retrieval in Direct Mode}

For streams requesting the rarely requested objects, their requested objects are serviced directly from disk and then delivered to the users(we call this type of data retrieval direct mode) with their own read ahead buffer. The very thing we must bear in mind is that as the number of disk streams increases, the aggregate disk bandwidth utilization increases and what is worse is that the amount of buffer memory increases extremely high as the disk utilization reaches $100 \%$. For more detailed information, how much read-ahead buffer is required with different numbers of concurrent streams, see $[5,10]$.

\subsection{Data Retrieval in Shared Mode}

For some streams accessing the same object, the data blocks existing between two streams are cached such that the following stream could avoid disk access until the end of the delivery(shared mode). In data retrieval in shared mode, when there is not so much free buffer space as new interval, interval changing occurs. In the existing interval caching algorithm[9], interval changing is made based only on the interval length of the new and existing intervals. But in our proposed caching scheme, the interval changing occurs only if the result of the interval changing reuires less buffer memory .

\subsection{Data Retrieval in Object Mode}

In the existing interval caching or buffer sharing policy, even if an object is rarely accessed, its interval between two consecutive streams will be cached provided that their interval is short enough to be cached. On the other hand, even if an object is accessed frequently, its interval between two consecutive streams will not be cached once their interval is too long to be cached. This means the existing interval caching policy does not consider the access patterns of the objects; it only considers the 
temporal locality in terms of request arrival. Therefore, in most cases, the buffered blocks are reused only once by the succeeding stream because not every object is requested so constantly such that all of their intervals are cached. So, if all blocks of some objects with high access frequency are cached entirely, its all blocks are cached as one logical interval.

\section{Experimental Results}

We assume that users' access patterns follow a Zipf distribution and all of the media objects are assumed to be MPEG II compressed with a playback rate of $8 \mathrm{Mbps}$. We also assumed that the size of media objects used in this simulation ranges from 10 to 30 minutes in terms of playback time.

The experiment is done only to check cache performance that the network resources are not taken into consideration. The proposed in the graphs means the proposed adaptive caching policy where two caching modes - shared and object - are applied together and eff is the caching policy, to which object mode is not applied to check the efficiency of shared mode caching. Finally, int is the existing caching policy proposed by [9]. As the experiment, buffer user ratio of three algorithms are checked and evaluated, with different arrival rates and buffer capacity. By buffer user ratio, it means how many streams out of all requested streams are serviced in the buffer cache, not by direct mode. As shown in Figure 3 and 4, the proposed algorithm shows higher buffer user ratio than other two algorithms except some cases; when the buffer cache is relatively small(600 MB) or the number of archived objects is large(1200). As the size of the buffer cache is large(2000 MB) and the streams' interarrival time is relatively long(say, $1.7 \mathrm{sec}$ ), three algorithms show not so much difference as in the case of short inter-arrival time( 0.43 or 0.56$)$. This is because, when the buffer size is large and the inter-arrival time is long, the buffer cache replacement does not occur so frequently that it is highly possible that once the object is cached, it could remain in the buffer cache as long as it could. The buffer user ratio also depends on the number of objects archived in the disks that as the number of objects archived gets larger, the buffer user ratio gets lower.

\section{Conclusion}

In this paper, we proposed a stream service strategy using the adaptive buffer sharing policy based on each object's access pattern. With the proposed policy, a steam is serviced one of three modes: direct, shared and object. The streams accessing rarely accessed objects are serviced directly from disks while active streams accessing the same object are serviced in shared mode where the interval between two streams is cached. Finally some objects accessed frequently are cached fully so as to fully avoid disk access. As a result of simulation, the proposed caching scheme is known to be more effective than other caching policies. This is because with the proposed algorithm, objects with less likely to be accessed are filtered as a function of its caching effectiveness by checking the requirement of read-ahead buffer memory and access frequency of objects archived. Moreover, some highly accessed objects are prefetched in their entirety so that unnecessary buffer replacement could be reduced. 

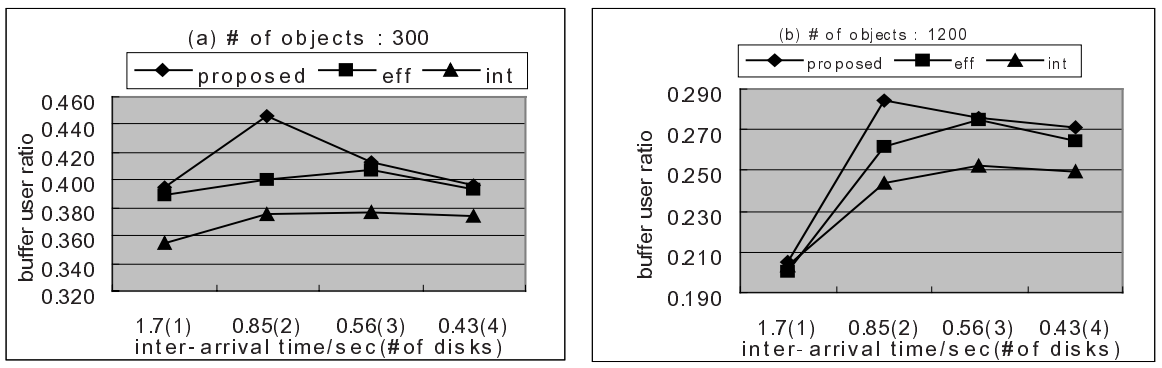

Fig. 1. Buffer user ratios when the buffer cache size is $600 \mathrm{MB}$
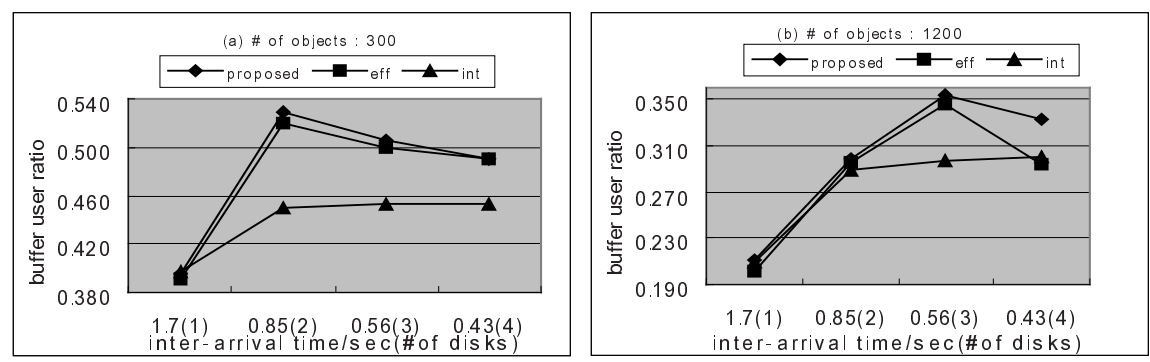

Fig. 2. Buffer user ratios when the buffer cache size is $2000 \mathrm{MB}$

\section{References}

1. Anderson D. P, Y. Osawa, and R. Govindan, “A File System for Continuous Media”, ACM Transactions on Computing Systems, Vol. 10, No. 4 pp. 311-337, Nov. 1992

2. A. Dan, D. Sitaram, and P. Shahabuddin, "Dynamic batching policies for an on-demand video server", Multimedia Systems, 4(3):112-121, Jun 1996.

3. A. Dan and D. Sitaram. "Multimedia Caching Strategy for Heterogeneous Application and Server Environments", IBM Research Report, RC 20670, Yorktown Heights, NY, 1996

4. Mon-Song Chen, Dilip D. Kandlur, P. Yu, "Optimization of the Grouped Sweeping Scheme(GSS) with Heterogeneous Multimedia Streams", Proc. ACM Multimedia 92 pp. 235-242, Anaheim CA, Aug, 1993

5. Kenchammana-Hosekote DR, Srivastava, "Scheduling Continuous media on a Video on Demand Server", In Proceedings of ACM Multimedia Computing and Systems, May 1994, Boston, Mass

6. Rangan P. V., H. M. Vin, and S. Ramanathan, "Designing an On-Demand Multimedia Service”, IEEE Communication Magazine pp. 56-65, Vol. 30 Jul. 1992

7. F. Tobagi, J. Pang, R. Baird, and M. Gang, "Streaming RAID - a disk array management system for video files", ACM Multimedia , pp 393-400, Aug. 1993

8. W. Shi and S. Ghandeharizade, "Trading Memory for Disk Bandwidth in Video On Demand Servers", In Proceeding of International Conference on Multimedia Computing and Systems, pp172-180, Jun 1998

9. R. Tewari, A. Dan, etal, "Buffering and Caching in Large-Scale Video Servers", In Proceedings of IEEE CompCon, 1995, pp. 217-224

10. Youjip Won, Jaideep Srivastava. "Minimizing Buffer Requirement for Continuous Media Servers, Multimedia Systems 8 (2000) 2, 105-117 